NOTA

$259-270$

\title{
BACON, ALGUNAS CONSIDERACIONES PRAGMÁTICAS DEL CONOCIMIENTO Y UNA METAFÍSICA ALTERADA ${ }^{1}$
}

Bacon, some pragmatic considerations of knowledge and an altered metaphysics

\author{
Alex Espinoza Verdejo*
}

\section{INTRODUCCIÓN}

La necesidad de analizar la obra Novum Organum (Bacon, 1949) es crucial para comprender los giros epistemológicos acontecidos en la historia del pensamiento. El experimentalismo de Francis Bacon es, en gran medida, el responsable de esta nueva propuesta. Bacon cambia la interpretación fundamentalista del mundo griego que abogaba por una actitud contemplativa de la verdad y con el único propósito de entender el mundo. En nuestro estudio veremos que Bacon tiene una inclinación especial por huir de los fundamentos con bases metafisicas, y privilegia en parte lo que Dewey llama pragmatismo (1954). Sin embargo, podremos también certificar que Bacon no es concluyente en su filosofía respecto de un abandono decidido de los constructos metafísicos.

\section{FRANCIS BACON Y EL PRAGMATISMO}

Existen ciertos tipos de afirmaciones que se heredan en la historia del pensamiento, una es la sostenida por Francis Bacon y expresada en su sentencia saber es poder. Esta afirmación representa el viraje en las ciencias ocurrida en la época moderna, la finalidad es lograr el dominio de la naturaleza por medio de las actividades desarrolladas por el conocimiento. En este sentido Bacon escribe:

La investigación de las formas que son (en razón cuando menos y conforme a su ley) eternas e inmutables, constituirá la metafísica; la investigación de la causa eficiente, de la materia, del progreso latente y de la constitución oculta (cosas todas que tienen relación con el curso ordinario y común de la naturaleza, y no con sus leyes fundamentales y eternas), constituirá la física (130).

Este testimonio de Bacon tendrá claras consecuencias en los estudios de la física y con ello, por ejemplo, se produjo la invención en el siglo XIX de la máquina a vapor siendo un factor determinante en la revolución industrial. Este era un anuncio del viraje epistémico, la inteligibilidad o comprensión profunda y metafísica de los fenómenos, como ideal griego, se modifica y se reemplaza por una interpretación novedosa del

\footnotetext{
${ }^{1}$ Este trabajo forma parte del Proyecto Mayor de Investigación de la Universidad de Tarapacá de Arica, Código 3730-15, del Convenio de Desempeño HACS de la Universidad de Tarapacá de Arica y del Proyecto Dicyt Código 031553DS de la Universidad de Santiago de Chile.
} 


\section{Alex Espinoza Verdejo}

conocimiento. De este modo, las ciencias modernas obtendrán una nueva significación: las ciencias deben ser útil a la humanidad. Estas ideas se plasman sin dificultad en la orientación filosófica del pragmatismo. Pierce al respecto afirma:

Una verdadera máxima lógica que yo he llamado pragmatismo es recomendada por mí por diferentes razones... Si solamente ella es verdadera, entonces es un instrumento maravilloso y eficaz. No únicamente para la filosofia. Yo he descubierto que ella rinde buenos servicios en todas las ramas de ciencia que yo he estudiado (263, mi Traducción).

También, mediante la palabra "poder" enunciada por Bacon, encontramos otras caracterizaciones propias del pragmatismo. Poder quiere decir dominio, control; dominio o control son demandas que provienen de las circunstancias, son estas las que definen y determinan el quehacer científico.

Todas estas apreciaciones de la filosofia de Bacon nos convocan a una lectura pragmática del conocimiento y esto se demuestra en el pensamiento de John Dewey, quien evoca las ideas de Charles S. Pierce, Dewey dice:

...Mr. Pierce después de indicar que nuestras creencias son realmente reglas para la acción, dice que para desarrollar el significado de un pensamiento necesitamos determinar qué conducta es adecuada para producirlo: tal conducta es para nosotros toda su significación (51).

Esta afirmación sugiere que el criterio de significación de cualquiera actividad humana, propuesto por Pierce, se interpreta bajo los efectos prácticos: la manipulación, las conductas. De lo anterior se desprende que la actividad científica no escapa a este nuevo rol que es esencialmente ejecutivo, dejando atrás la búsqueda de las causas finales, el mismo Pierce lo reafirma:

Por otra parte, puesto que es imposible saber intuitivamente que una cognición dada no está determinada por un conocimiento anterior, la única forma de saberlo, es por inferencia hipotética a partir de los hechos observados (33, mi traducción).

Así, las ciencias modernas están gobernadas por el ideal de la eficiencia, lo central es determinar el modo de cómo los fenómenos se comportan cuando los relacionamos con nuevas variables. El experimentalismo es una consecuencia obligada que se desprende de lo anterior y la actividad científica se define esencialmente en ella. Las discusiones científicas se centran en los logros provocados por los mecanismos de control de las variables, más que en sus alcances y logros teóricos, en otras palabras, la ciencia consiste principalmente en establecer leyes con el fin de predecir, de determinar los estados futuros de los sistemas naturales. El renacimiento nos ha dejado como legado, para el desarrollo ulterior de la ciencia, el patrocinado olvidadizo de las causas últimas para conquistar el grado epistémico más profundo: el entendimiento. 
Estas propuestas se sellan en el antiaristotelismo de Bacon, publicitado en su obra Novum Organum cuyo objetivo principal es promover la idea que la ciencia debe ocuparse de las causas eficientes:

Se establece también que hay cuatro especies de causas: la materia, la forma, la causa eficiente y la final. Pero dista tanto la causa final de servir a las ciencias, que más bien las corrompe, a menos que se estudie las acciones del hombre. El descubrimiento de la forma es considerado como imposible (11).

Las palabras de Bacon son indicadores de la actitud antiaristotélica y antimetafísica, asumiéndose la ya menciona interpretación pragmática de la ciencia. Las señales del pensamiento renacentista indican que la actividad científica debe poner especial cuidado en las cuestiones metodológicas.

La interpretación pragmática de las ciencias se refleja en las condiciones que debe cumplir la implementación de una situación experimental; condición que exige un cuidado único en la elaboración de los componentes experimentales, de allí que las manipulaciones de las variables sea un tema crucial:

Mas el verdadero método de la experiencia, por el contrario, primero enciende la luz, y luego por medio de la luz señala el camino empezando por una experiencia ordenada y madura y no desordenada y al azar, y deduciendo de ella los axiomas, y de los axiomas así establecidos otra vez nuevos experimentos; pues ni el verbo divino operó sin orden sobre la masa de las cosas (Bacon, 131).

La situación en la que se constituye el experimento es esencial para determinar y definir la verdad de manera "madura", no se deja cabida para el azar, la idea es acortar espacio a lo inesperado.

\section{BACON, LA CERTEZA Y LA INTERPRETACIÓN PRAGMÁTICA DEL EXPERIMENTALISMO}

La influencia que tuvo el pragmatismo en el conocimiento científico en una época de profundos cambios epistémicos, no excluyeron el gran ideal de verdad representado en la certeza. En este momento histórico siglo XVII no vemos en ciencia una participación decidida del concepto de probabilidad, ya sea en el sentido objetivo o subjetivo $^{2}$. Sabemos que Bacon siente una admiración especial por los descubrimientos realizados por Galileo y que esta admiración depende de la exactitud en las predicciones, no se acepta el error y con ello el tema de las probabilidades es irrelevante.

A lo anterior podemos agregar que el método baconiano se define como un método que nos aísla de las experiencias erróneas. Muchas de las experiencias erróneas provenían de los dogmas ( $\delta \dot{\gamma} \gamma \mu \alpha)$ establecidos por las tradiciones, como era el caso del empirismo radical o del racionalismo. Así, por un lado, advierte Bacon, el empirismo radical debería aceptar la participación de verdades probables, cuando se acepta la

\footnotetext{
${ }^{2}$ La probabilidad subjetiva basa la predicción en hechos ya acontecidos; en cambio la objetiva predice de acuerdo a las situaciones iniciales, por ejemplo obtener un as en el lanzamiento de un dado es de probabilidad 1/6.
} 


\section{Alex Espinoza Verdejo}

inducción por enumeración como fruto de las constataciones empíricas inmediatas. Por otro lado, el racionalismo corre el riesgo de caer en una especie de absolutismo, ya que se exige que los conceptos, utilizados en una investigación, deban estar bien definidos, en este sentido el nominalismo es una especie de idealismo, concepción filosófica públicamente rechazada por Bacon. Para evitar de caer en este error, Bacon sugiere que toda investigación debe someterse al análisis de la idola del forum, es decir, se debe tener cuidado en no abusar del uso del lenguaje, que en definitiva es lo que da vida a las teorías. Para Bacon el experimentalismo será el arma de lucha en contra de los dogmas que pretenden alejarse de la exactitud y de la claridad como componentes esenciales de la cultura de la investigación científica, Boutroux en su libro L'idée de loi naturelle, manifiesta:

Los creadores de la filosofia moderna, Bacon y Descartes, han determinado que el objetivo de la ciencia es descubrir leyes y que estas deben cumplir con un criterio doble: ser universales y que se refieran a una realidad determinada. Esta idea sobrepasa el punto de vista antiguo para quien las leyes deben ser generales e ideales, yendo más allá de lo verdadero, de lo posible, y así se persigue conocer lo real de manera absoluta y precisa... Pero siendo el mismo objetivo para ambos pensadores, los medios que ellos emplean son diferentes: Bacon sigue la dirección empirista, Descartes la dirección racionalista (6-7, mi traducción).

Frente a estos dos enfoques: empirismo radical y racionalismo, el pragmatismo, representado en el experimentalismo, se ofrece como una alternativa válida. La idea de fondo ya no es entender por medio de una la verdad absoluta única y perfecta, como lo sugirió Descartes: ideas claras y distintas, sino que la verdad se define como acción útil con carácter de ser beneficiosa para el ser humano. De allí que el concepto de poder, de dominio, son cualidades necesarias para dar respuestas a la contingencia, pero esto es contradictorio con la idea que sostiene la cultura griega clásica en cuanto la verdad constituye un goce estético por parte del espíritu. El pragmatismo está más cerca del sensualismo y de una actitud hedonista frente al mundo. Esto es una consecuencia natural del poder predictivo y de la certeza que propicia el método experimental.

La interpretación pragmática del experimentalismo define el método como una entidad dinámica cuyo objetivo es determinar el paso de los efectos a las causas. Así nuestro autor revela su predilección por la inferencia inductiva, es decir, pasar de los efectos a las causas, ir a la búsqueda de los axiomas mayores. La sugerencia de Bacon es comenzar desde los mismos hechos existentes en una temporalidad y espacialidad singular y una vez establecidos los axiomas mayores, podemos construir sistemas deductivos que permitirán realizar predicciones exactas. Así, el método baconiano fundará lo que hoy llamamos modelo explicativo hipotético nomológico deductivo ${ }^{3}$. Él dice que debemos atenernos a la idea de buscar los axiomas verdaderos

\footnotetext{
${ }^{3}$ Las ideas positivistas en ciencia harán que las leyes tengan un carácter de hipótesis pero que se configuren como leyes en la medida que ellas permitan la deducción de consecuencias demostrables. Esta idea es la que propone Carl Hempel en su libro Filosofia de la ciencia natural.
} 
representados en los llamados esquemas (schemes) o formas, que serían los responsables de determinar el conocimiento dándole un carácter certero y no como verdades probables:

Este proceso latente del que estoy hablando, es algo muy distinto de lo que puede ocurrir a las mentes de los hombres, llenas de preocupaciones como están ahora. Pues por él no entiendo ciertas medidas, o signos o escalas de progreso, visibles en los cuerpos, sino más bien un proceso continuado que en su mayor parte escapa a los sentidos (Bacon, 179).

El proceso latente es lo que constituye el esquema del fenómeno, los esquemas son captados gradualmente gracias al trabajo de depuración de los análisis experimentales exhaustivos, así estarían liberadas de los prejuicios y de los errores, revelándose así la certeza en toda plenitud.

Si bien es cierto que el concepto de probabilidad no es preponderante en ciencias, sin embargo podemos decir que en el siglo XVII el concepto de probabilidad recién se comienza a notar. En el pensamiento baconiano no hay espacio para este concepto, no hay necesidad de un concepto matemático de probabilidad, ni de un concepto cualitativo de él. La razón fundamental es que el modelo de ciencia es aún la geometría, la mecánica y la astronomía, todas ellas son ciencias racionales y demandantes de la exactitud aplicada al análisis de los fenómenos naturales. Para asignar un lugar al concepto de probabilidad en las ciencias, es necesario extender el concepto de ciencia de manera que se pueda aplicar al estudio de fenómenos aleatorios.

Bajo este orden de ideas podría pensarse que Bacon trataría el concepto de probabilidad cuando este se evoca al estudio de la inducción, sobre todo cuando piensa en la inducción por enumeración, es decir, que la simple enumeración de objetos A1, A2, A3...que tienen la propiedad B se concluiría que todos los A son B. Bacon se aleja de este tipo de inducción, porque este tipo de inducción abrigaría el concepto de probabilidad, es más, Bacon no veía un uso adecuado de este tipo de razonamiento por encontrarlo muy elemental y poco seguro para aventurar una verdad exacta a futuro, de este modo afirma:

Pues la inducción que procede por enumeración simple es una cosa pueril, sus conclusiones son precarias y expuestas al peligro de un contradictorio y las más de las veces deciden por un número de hechos menor de lo debido y por solo aquellos que están a la mano (145).

Bacon quería ir mucho más allá de los datos empíricos, él quiere una construcción abstracta de un mundo posible, y es esto lo que él llamará induction vera, fundamento último de su experimentalismo: el conocimiento se origina en el mundo circunstancial físico y este mismo mundo puede proyectarse a otros mundos posibles dotados de exactitudes proporcionados por el mismo método.

Sin embargo, estas ideas nos sorprenden porque tanto la exactitud como los esquemas son nociones que encontramos en el vocabulario metafísico. Bacon busca 
alejarse de la aristotelia, pero estos conceptos más bien lo acercan, esto hace dudar del Bacon que estamos habituados, vale decir, de un Bacon antimetafísico y antiaristotélico.

\section{BACON, LAS MATEMÁTICAS Y LA EXACTITUD}

Para seguir en la misma línea de pensamiento, el apego incondicional a la empiria sostenido por Bacon le impidió reconocer el valor de las matemáticas. Su punto de partida no es el mismo que el de los pitagóricos: creer que la belleza de la naturaleza se debe a su estructura matemática. Si bien es cierto que Leonardo da Vinci, Kepler, Galileo, Newton se representan bien en las ideas del experimentalismo de Bacon, pero no es menos cierto que estos sabios también han adoptado el ideal Pitagórico: creer que la naturaleza es cognoscible, porque ella está matemáticamente estructurada y determinada, es decir, es medible ${ }^{4}$.

Estos dos tipos de conocimiento, ya sea empírico o racional, ponen en el escenario de la discusión el concepto de inducción, logrando un cambio en su significado, no se trata de obtener una conclusión a partir de la enumeración de los casos favorables a una idea. Tampoco se trata de obtener una teoría abstracta a partir de un listado de datos empíricos: se trata ahora de encontrar las relaciones matemáticas que permanecen inalterables en la serie de fenómenos determinados por la medida, y cuyo objeto de estudio es el movimiento. La inducción en Galileo, Kepler y Newton se caracterizan por: encontrar las relaciones matemáticas invariables, por determinar los fenómenos por las medidas en la investigación del movimiento.

Así, vemos que detrás de los fenómenos existe una especie de ontología implícita, lo que existe es el movimiento y la manera de representarlo es mediante las relaciones matemática, existe en ello una correspondencia entre el lenguaje matemático y la realidad.

La teoría matemática del movimiento o la mecánica ha sido elaborada por Galileo, este sabio relacionó la fuerza con la masa y la aceleración, esto fue posible gracias a estos presupuestos ontológicos y epistemológicos. Allí en donde la inducción de Bacon busca las formas, y finalmente las formas simples, especies de átomos o de cualidades primarias, Galileo con el método de resolución, busca los procesos descritos por el movimiento determinado matemáticamente. Entonces, por un lado, allí donde la induction vera de Bacon consiste en estudiar cómo los fenómenos se relacionan para formar una estructura empírica, por otro lado, la síntesis de Galileo, o el modo de composición, se dedica a demostrar que los resultados de la teoría matemática son adecuados a los contenidos de experiencia.

Así como notamos un cambio en el concepto de inducción, también notamos que existe un cambio en el concepto de experiencia, sobre todo cuando pasamos de

\footnotetext{
${ }^{4}$ El ideal pitagórico lo encontramos vigente hasta nuestros días, un claro ejemplo es la teoría de la relatividad. Ella pone muestra de cómo por medio del cálculo podemos dar cuenta de fenómenos que no son perceptibles y que no son manipulables físicamente (Espinoza, 167)
} 
Bacon a Galileo. Para Bacon una experiencia científica es una cuestión precisa propuesta a la naturaleza para atrapar una ontología definida; para Galileo, en cambio, implica una intervención en los fenómenos para someterlos a medición, existe un predominio de las determinantes epistemológicas. Galileo ha integrado un nuevo antecedente al método experimental de Bacon, los fenómenos naturales deben matematizarse. La garantía que brinda el uso de las matemáticas para explicar los fenómenos naturales se fundan en la búsqueda de la verdad representada en la exactitud. Esta concepción del método científico de Galileo ha sido utilizada exitosamente más tarde en la mecánica newtoniana ${ }^{5}$.

\section{BACON, ACEPTACIÓN Y RECHAZO DE LOS SUPUESTOS METAFÍSICOS}

Para extender la reflexión acerca de las consideraciones ontológicas en el experimentalismo de Francis Bacon, debemos hacer mención del concepto de esquematismo latente, concepto de nuestro autor y que ya hemos enunciado:

Este proceso latente del que estoy hablando, es algo muy distinto de lo que puede ocurrir a las mentes de los hombres, llenas de preocupaciones como están ahora. Pues por él no entiendo ciertas medidas, o signos o escalas de progreso, visibles en los cuerpos, sino más bien un proceso continuado que en su mayor parte escapa a los sentidos (179).

Esta noción no corresponde, según él, a una forma de expresión de cómo nos referimos a las cosas, tampoco corresponde a los contenidos de información brindada por los sentidos. Según Bacon, la materia estaría compuesta por partículas con configuraciones específicas: schematismi, teniendo también la cualidad de autoorganizarse: meta-schematismi, esta autoorganización de la materia significa que ella tiene la particularidad de recrear su configuración. Los schematismi son elementos invisibles al ojo desnudo, ellos son elementos esenciales de la materia, los schematismi permiten la identificación y transformación de las cosas. En este sentido, la ontología baconiana escapa al control físico y esto confunde a un lector que quiere ver a Bacon como un pensador estrictamente empirista.

Desde una perspectiva ontológica los schematismi se representan en los axiomas superiores y estos necesitan del control empírico con la finalidad de no sobrepasar la interpretación, es decir, para que la actividad del entendimiento gobernada por el silogismo no vaya más allá de los hechos. En consecuencia, sugiere Bacon, la tarea principal del método experimental es determinar los axiomas medios, esto se facilita

\footnotetext{
${ }^{5}$ Pierre Duheme en su libro La théorie physique son objet- sa structure, y en el capítulo de Qu'une loi de physique n'est, a proprement parler, ni vraie ni fausse, aprrochée, sugiere que las leyes científicas nos proporcionan una verdad aproximativa de la realidad, porque ellas son representaciones simbólicas y que los simbolismos son formas de explicar dependientes de los marcos teóricos y estos son cambiantes. Frente a este problema el experimentalismo escapa de estos problemas, puesto que Bacon siempre promueve la idea que los hechos deben hablar por sí mismos
} 
una vez que se haya estabilizado la conexión de los hechos con la idea representada en el mundo de los entes abstractos. La conexión de los dos mundos depende de la calidad de la organización de los sistemas que se encuentran en la interacción observacional. Sabemos bien que para Bacon la calidad de la observación depende del experimento. Esto es lo que avala el éxito del saber, vale decir, el saber que se transforma en poder.

El saber exitoso es una consecuencia de la herencia experimentalista, y se transforma en poder en la medida que la ciencia esté apta para predecir los estados futuros o pasados del sistema. Estos argumentos caracterizan muy bien lo que se entiende por el pragmatismo en las ciencias. Esto se refleja en la insistencia de Bacon por la oposición a la filosofía de Aristóteles; oposición que se representa en sus esfuerzos con el fin de determinar las causas eficientes de los sistemas; causas que se pueden controlar de manera experimental, la causa final es impropia: "De allí que la causa final esté fuera de los dominios de las ciencias, ella corrompe, salvo en el caso de las ciencias humanas" (Bacon, 172).

La afirmación anterior sostenida por Bacon nos lleva a pensar en la predilección por las ciencias fisicas, ella cumple con los mandatos del experimentalismo, no olvidemos que su filosofia es producto del desarrollo de la física de Galileo. Sin embargo, percibimos, de igual forma, ciertos problemas que el mismo Bacon ha tratado en su obra; problemas que se originan cuando se pretende justificar la relación entre las causas eficientes y sus generalizaciones. Estas ideas se estudian en el aforismo XIX del Novum Organum que trata el tema de la imposibilidad de pasar de los hechos a los axiomas generales. La recomendación baconiana se define en la gradualidad. Bacon precisa que el paso de los hechos particulares a los axiomas generales se debe realizar de manera gradual con la ayuda y asesoría del juego dialéctico representado en el método experimental; se inicia con las ontologías preformadas, es decir, con los datos adquiridos por los órganos de los sentidos, luego será necesaria la induction vera, la que determine y defina una ontología. Es evidente que esto manifiesta, una vez más, el antiaristotelismo de Bacon, vale decir, desconfía del conocimiento fundado en la intuición, porque ella escapa de cualquier tipo de control:

Así, por consiguiente, yo no doy mucha importancia a las percepciones inmediatas que son propias de los sentidos, sino que yo llevo la cosa al punto donde el sentido será el único jurado de la experiencia y de la experiencia de la cosa (Bacon, 56).

Con esta cita queda claro que nuestro autor no acepta la inmediatez del conocimiento, el camino hacia los axiomas generales parte de los datos sensible, pero esto es poco claro: ¿cómo puede el sentido ser jurado frente a lo que se siente? Con estos antecedentes Bacon pretende escapar del aristotelismo y de los constructos teóricos-metafísicos, pero esta decisión es poco convincente, porque la única forma de encontrar una solución al tema del origen del conocimiento, y con ello a la inducción, es recurriendo a antecedentes metafísicos, Aristóteles propone la intuición. Esta propuesta 
del estagirita deja la inducción como una inferencia válida y con ello queda garantizada la construcción del conocimiento.

\section{BACON Y LA METAFÍSICA ARISTOTÉLICA}

El experimentalismo de Bacon acepta el canon empirista de la repetitividad respaldada por la induction vera y de la observabilidad, y así se aleja de los aires impregnados de teleología que cubrían otrora el pensamiento, sin embargo no podemos olvidarnos que Aristóteles también acepta la repetitividad, la divergencia se radicaliza en la consideración que tiene Aristóteles por la intuición como conocimiento inmediato, factor determinante en la elaboración de las premisas universales, elemento esencial de la silogística:

Teniendo en cuenta estas consideraciones, podemos deducir que no podrá haber conocimiento científico de las premisas primeras, excepto las propuestas por la intuición, nada será más verdadero que el conocimiento científico, la intuición será encargada de aprehender las premisas primeras, conclusión que emana del hecho que la demostración no puede ser la fuente originaria de la demostración no más que el conocimiento científico no puede ser la fuente del conocimiento científico.

Frente a estos temas, Bacon deberá competir con la metafísica del estagirita y buscar la infalibilidad en la induction vera y esta se encuentra en las relaciones ontológicas naturales, en Bacon el antisustancialismo aristotélico es evidente. Sin embargo, es fácil darse cuenta que el discurso de Bacon no escapa de las consideraciones sustancialitas en su sistema filosófico, da curiosamente el nombre de forma, como ya lo hemos dicho anteriormente, a las verdades encontradas en los resultados de la experimentación. Lo que suscita la curiosidad es el significado que la historia ha atribuido al concepto de forma. Sabemos bien que Bacon no acepta la interpretación aristotélica, la forma no es equivalente a una especie de esencia que está implicada en las cosas mismas y que determina la identidad específica, la causa formal para Aristóteles tiene esa connotación metafísica, es lo que determina la clase. Bacon, proporciona una nueva connotación al concepto de forma, la forma se interpreta como figura. Así predominará la idea en que la figura representa a la descripción de los sucesos naturales que tienen el carácter de ley, y que además se desenvuelven a través de las coordenadas de tiempo y espacio ${ }^{6}$.

En otra época, la filosofía de John Stuart Mill no escapó de estos temas, él prefiere hablar de uniformidad en la naturaleza, según este pensador, en la naturaleza existen regularidades, y cada una de ella representan fenómenos particulares, no existe

\footnotetext{
${ }^{6}$ Según André Lalande, "Las palabras forma y especies corresponden al eidos de Aristóteles. Se puede afirmar que existe una división del sentido de eidos, entre estos dos términos, forma representando al eidos en el sentido de carácter común, y especies representando al eidos en el sentido de especie o clase constituida por la posesión de este carácter común" (Traducción mía).
} 
una gran regularidad desde la que fluyan el resto, afirma que las inducciones más fuertes apoyan a las más débiles, cuando esto último ocurre, entonces las inducciones fuertes tendrán el carácter de ley natural, Mill representa claramente la idea de Bacon, en este sentido explicar es dar cuenta de la red de causas eficientes, la causas finales están completamente fuera de estos dominios. Mill piensa que la manera de alcanzar la generalización de las regularidades naturales es mediante la matemática (252).

\section{BACON Y EL NOMINALISMO}

Así, por un lado, podemos interpretar empíricamente el concepto de ley, en la medida que la naturaleza guarda un grado de confirmación como medio probatorio de los eventos. Por otro lado, podemos afirmar que el concepto de ley obedece a los cánones del nominalismo, vale decir, la universalización de las leyes naturales descansarían en vocablos que han prosperado al filo del tiempo. Bacon estaría dispuesto a aceptar que la convergencia entre el ser y el conocer descansa en las operatorias cognitivas representadas en la induction vera, esta última no podría configurarse sin el respaldo del nominalismo, el método experimental necesita que las definiciones conceptuales de las variables sometidas a su juego dialéctico sean las más claras posibles y que estén fuera del alcance de las interpretaciones. Sin embargo, existen afirmaciones de Bacon que nos llevan a confusiones como es el caso cuando él afirma que el experimentalismo tiene por finalidad eliminar las idolas, una de ellas es la idola del fórum, con ello se pretende poner fin a los problemas suscitados por el lenguaje ${ }^{7}$.

La posición antinominalista recién descrita se pone en duda, porque la situación experimental necesita de una construcción artificial o teórica representada por el mismo método, Bacon afirma:

La mano desnuda o el entendimiento abandonado a él mismo no tiene un gran poder, la cosa se hace gracias a los instrumentos y a los apoyos que son tan necesarios a la inteligencia como a la mano; y de la misma manera que los instrumentos de la mano determinan o guían sus movimientos, aquellos del espíritu inspiran el intelecto o lo previenen (72).

Este párrafo demuestra la importancia que adquiere el plan previo en una situación experimental, con la finalidad de llegar a la comprobación de los supuestos hipotético-teóricos, siendo estos últimos, necesariamente, construcciones lingüísticas, producto de la inspiración intelectual.

Es así como Bacon manifiesta la idea de reorientar el camino para la conquista de un verdadero conocimiento, para ello se hace necesaria la utilización de un método

\footnotetext{
${ }^{7}$ Bacon tiene en mente el nominalismo medieval que distingue el ser del conocer. Cuando usamos un nombre no significa que haya una propiedad real universal e idéntica compartida por los objetos a los cuales el nombre común se aplica. El nombre de una cosa es una entidad vacía de realidad, existen las cosas, pero solo conocemos sus nombres. Esto último marca la distinción entre el ser y el conocer, ideas muy bien aceptadas por el nominalismo hobbesiano.
} 
que depure y que controle el conocimiento vulgar. Por esta razón Bacon propone un sistema inductivo que satisface los criterios de rigor y de sistematicidad, además se esfuerza por probar la verdad de un hecho particular sometiéndolos a operaciones minuciosas y controladas para conquistar los axiomas mayores. Así, uno está atento a las redes de ontologías reales determinadas por las coordenadas del tiempo y del espacio, y a su propia trascendencia para esperar no solamente la generalización, sino que también, y sobre todo, el descubrimiento y la predicción. Una vez más, el pragmatismo justifica la inducción baconiana, la utilidad de las ciencias depende del nivel de logros de los cálculos, la exactitud es lo preponderante, estas exactitudes son realizables en la medida que los campos ontológicos estén representados en sistemas lingüísticos bien definidos, cada variable que participe en la situación experimental debe ser bien delimitada.

\section{CONCLUSIÓN}

La historia de la evolución del conocimiento ha sufrido infinitos cambios, muchos de ellos representan alteraciones de matices, pero el cambio más profundo se representa en el legado del experimentalismo de Francis Bacon. Con ello se ha atentado y fracturado decididamente una concepción que podríamos denominar romántica del conocimiento, es decir, conquistar la verdad con la única intención de comprender el mundo en donde realizamos nuestras vidas, esta había sido la opción de los sabios griegos. La intelección era el objetivo de la racionalidad temprana, estar en posición de la verdad no es más que entrar al juego de la admiración, del desinterés material. Con la conquista de la verdad lograda por el intelecto era en el fondo la realización, la felicidad ( $\varepsilon v \delta \alpha u o v i \alpha)$. Algunos de los grandes sabios de nuestra época, como es el bien sabido caso de Einstein, pensaban que el mundo posee explicaciones simples y que los poderes de las matemáticas podrían alcanzar la simpleza del universo. Bacon no fue más allá del juego físico de la relación entre las cosas, Bacon no matematizó el mundo, de ahí que su experimentalismo ofrece una visión de mundo fundado en el pragma, en la acción. El conocimiento se resuelve en la relación y en su éxito, es esto lo que define la verdad, el éxito en ciencia se determina por el poder predictivo de los sistemas. Así, Bacon no admitirá interpretaciones probabilísticas en los resultados de sus investigaciones experimentales, la induction vera debe ser infalible.

El advenimiento del desarrollo de una sociedad, de una Inglaterra fundada en el capital, en el poder material, hizo que el experimentalismo tuviera sus momentos de gloria, y esto ha trascendido todas las fronteras del conocimiento hasta nuestros días. Hoy las ciencias han podido conquistar el conocimiento bajo la integración de las matemáticas con los hechos expresados en los experimentos, el resultado ha sido el positivismo. La ciencia ha continuado rindiendo satisfacciones a la evolución humana, esto último se debe, en gran parte, al desarrollo acelerado de la tecnología, condición que consolida el pragmatismo. Como consecuencia de lo anterior la ideología filosófica del realismo científico seguirá dominando los espíritus de la modernidad. El realismo 
metafísico, realismo que piensa que la naturaleza está compuesta de una metafísica implícita, cuya exigencia es la intelección, no es la ideología requerida por Bacon.

Como hemos analizado, Francis Bacon ha sido el precursor de esta nueva tendencia que ha marcado el desarrollo de las ciencias, sin embargo, también hemos analizado que el sistema baconiano si bien se autodefine como antiaristotélico, queda en la incertidumbre cuando se quiere encontrar el fundamento último del conocimiento. Bacon intentó, bajo una tímida actitud, reencontrarse con fundamentos metafísicos porque de otra forma sus propuestas corrían el riesgo de caer en el escepticismo.

En la obra Novum Organum de Bacon se dibuja la pretensión humana de alcanzar los axiomas mayores, el temor se acentúa una vez que no admite la participación de la interpretation en la lectura de los hechos naturales, él quiere que los hechos hablen por sí mismos. Negar la intuición, negar las formas, negar la existencia de las causas últimas, y entender este mundo natural como un sistema entramado de causas eficientes, conducirán a Bacon, y a la ciencia de nuestra época contemporánea, a permanecer en un sitial relativista. Las verdades eternas no existen, las ciencias deberán conformarse con el relativismo pragmático.

Universidad de Tarapacá* Avda. 18 de Septiembre 2222. Arica (Chile)

aespinoz@uta.cl

OBRAS CITADAS

Aristóteles. Obras, Madrid, Edit. Aguilar, 1977.

—_ Lógica. Analíticos, Post. Libro II. Cap. 19, Madrid: Aguilar, 1977.

Bacon, Francis. Novum Organum, Buenos Aires: Losada, 1949.

Boutroux, Emile. De l'idée de loi naturelle, Paris: J.Vrin, 1925.

Dewey, John. Pragmatismo, Argentina: Aguilar, 1954.

Duheme, Pierre. La théorie physique son objet- sa structure, Paris: J. Vrin, 1981.

Espinoza, Miguel. Théorie du Déterminisme causal, Paris: L'Harmattan, 2006.

Lalande, André. Vocabulaire Technique et critique de la philosophie, Paris: Puf, 1988.

Mill, John Stuart. Système de logique, Bruselas: Pierre Mardaga, 1988.

Peirce, Charles Sanders. Pragmatisme et pragmaticisme, París: Du Cerf, 2002. 